FEDSM2017-69167

\title{
PARAMETRIZATION OF HIGH-SPEED TRAIN STREAMLINE SHAPE
}

\author{
Zhenxu SUN \\ Key Laboratory for Mechanics in Fluid Solid \\ Coupling Systems, Institute of Mechanics, \\ Chinese Academy of Sciences \\ Beijing, China \\ Guowei YANG \\ Key Laboratory for Mechanics in Fluid Solid \\ Coupling Systems, Institute of Mechanics, \\ Chinese Academy of Sciences \\ Beijing, China
}

\author{
Ye ZHANG \\ Key Laboratory for Mechanics in Fluid Solid \\ Coupling Systems, Institute of Mechanics, \\ Chinese Academy of Sciences \\ Beijing, China
}

\section{ABSTRACT}

In the past decade, the high speed trains (HSTs) in China have experienced a booming development, with the design of CRH380A as a predominant example. A series of brand new HSTs have been developed with high aerodynamic performance, which includes the running resistance, the lift of the trailing car, pressure waves when trains pass by each other, aerodynamic noise in the far field, etc. In order to design HSTs with better aerodynamic performance, it is necessary to perform aerodynamic shape optimization, especially to optimize the streamline shape of HSTs. Parametrization is the basis for the whole optimization process, since good parametrization approach not only affects the optimization strategy, but also determines the design space and optimization efficiency. In the present paper, a series of work related to the streamline shape parametrization performed by the author in recent years have been introduced. Four different parametrization approaches have been exhibited, which are Local Shape Function method (LSF) and Free-Foam Deformation method (FFD), Modified Vehicle Modeling Function method (MVMF), Class function/Shape function Transformation method (CST). These methods could be categorized into two kinds: shape disturbance approach (LSF and FFD) and shape description approach (MVMF and CST). Among these four methods, some are developed by the authors while some are locally modified so as to meet the parametrization of the streamline shape. The detailed process of these four approaches are exhibited in the present paper and the characteristics of these four approaches are compared.

\section{INTRODUCTION}

In the past decade, the HSTs in China have experienced a booming development, with the design of CRH380A as a predominant example. The operation speed reaches up to 300 $\mathrm{km} / \mathrm{h}$ and the biggest experimental speed reaches to a value of $500 \mathrm{~km} / \mathrm{h}$. With a big slenderness ratio, HSTs run close to the ground with a high speed. As a result, many aerodynamic issues which could be neglected in low running speed circumstance become to be dominant when the speed rises $[1,2]$, such as the aerodynamic resistance, aerodynamic lift of the trailing car, pressure waves, micro pressure waves and aerodynamic noise. Current study reveals that the aerodynamic loads of the leading and trailing cars have the greatest impact on the safety and stability of the train operation. The head shape of the highspeed train has great influence on the aerodynamic performance of the train. Aerodynamic performance of HSTs could be greatly improved by properly designing the streamline shape [36].

The HSTs with operation speed over $300 \mathrm{~km} / \mathrm{h}$ mainly include the Shinkansen trains from Japan, the ICE3 train from Germany, the TGV trains from France and the CRH380 series from China, which are shown in Figure 1. The streamline shapes vary significantly from different series. However, the starting point for designing a streamline shape is always to improve the aerodynamic performance in some aspect. Meanwhile, the train body processing technology and the cultural characteristics are also considered. The slenderness 
ratio for Shinkansen trains is comparatively larger, which is mainly to reduce the pressure waves and micro-pressure waves when trains pass the tunnel. The slenderness ratios for ICE3 and TGV series are comparatively smaller, which is mainly to reduce the aerodynamic drag and balance the processing requirement. As one of the representatives of the new generation of HSTs in China, the CRH380A train is innovated independently on the base of E2 series, and the aerodynamic performance, cultural characteristics and the processing technology are all considered during the design of the streamline shape.

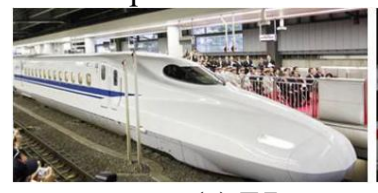

(a) E7

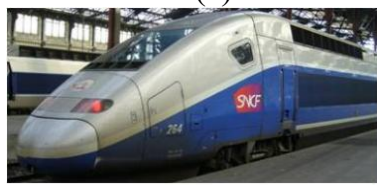

(c) TGV

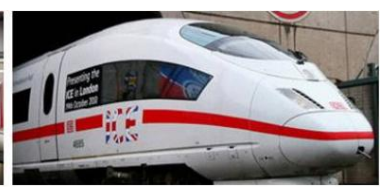

(b) ICE3

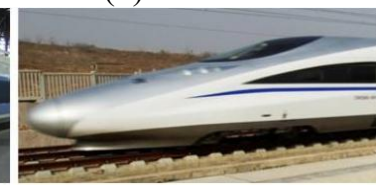

(d) CRH380A
Figure 1. Different kinds of HST streamline shapes: (a) E7; (b) ICE3; (c) TGV; (d) CRH380A.

In order to improve the design efficiency and shorten the design cost, aerodynamic optimization on the base of optimization algorithms becomes a research hotspot for the design of streamline shape [7, 8 and 9]. For three-dimensional aerodynamic optimization of streamline shape, parametrization is the basis of the whole study.

Due to the big slenderness ratio, the streamline shape of HSTs is composed of complex free-form surfaces. During the optimization process, the surface deformation could be rather large, which would generate totally different kind of surfaces. As a result, it is very crucial to develop parametrization methods for the streamline shape of HSTs. Current frequently used parametrization methods for three-dimensional surfaces include mesh vertex method, free-form deformation method (FFD), spline surface method and analytical method. Mesh vertex method could describe arbitrary shape and is widely used in actual streamline shape parametrization [10-13]. However, non-smooth surface could be easily generated and this approach suffers greatly from the number of design variables and the gradient between adjacent vertices. FFD [14] and spline surface method show good performance in shape parametrization, which can control the large deformation area with fewer control points, and can ensure the smoothness of the surface. Analytical method is widely used for those simplified shapes $[9,15$ and 16], which get deterministic equations and could be easily modified by varying the coefficients of the equations. As a result, it is difficult to use in parameterizing practical streamline shape.

Parametrization is the basis for the whole optimization process, since good parametrization methods cannot only fully describe the shape deformation, but also shorten the optimization period to a certain extent and improve the optimization efficiency. This paper focuses on the parametrization methods for the streamline shape of HSTs, and introduces four different kinds of parametrization methods developed by the authors. The exact process of these methods and their applications on HSTs are mainly focused on, and also their performances is compared.

\section{SHAPE DISTURBANCE METHODS}

The definition of shape disturbance method is first given here. These methods aim at an already-existing shape, and achieve new shape by the superposition of the original shape and increment, which is generated by the disturbance of different design variables along the original surface. Shape disturbance method usually benefits from high deformation efficiency and stronger applicability. In this section, two parametrization methods are introduced, which are Local Shape Function method and Free-Form Deformation method.

\subsection{LOCAL SHAPE FUNCTION METHOD}

LSF method was first proposed by the authors [17], and the whole processes are as follows:

(1) For a given geometry, deformation regions should be divided firstly.

(2) Mesh the deformation regions, and obtain the coordinate values of every grid point. In order to keep the smooth transition of the surface, the structural grids have been utilized for mesh discretization, just as Figure 2 shows.

(3) Choose the deformation function of each region, which can be selected randomly, but smooth transition between adjacent regions should be ensured.

(4) Choose a weight factor $w_{i}$ for each deformation function, which determines the maximum deformation value of each region.

(5) Calculate the increments $\Delta$ of coordinates of all grid points by the deformation functions and $w_{i}$.

(6) Get the coordinates of the deformed shape by summing $\Delta$ and the coordinates of the original shape.

(7) According to the coordinates of the deformed shape, the deformed surface can be fitted exactly, then a deformation process is done.

In the above process, step (3) is the most crucial step. A control point is assigned on each deformation region, which could be used to deform the surfaces by the movement of control points. The deformation surfaces are different from each other due to the different choices of deformation functions. Inappropriate deformation functions will easily lead to irrational deformation surfaces. Trigonometric functions, exponential functions, logarithmic functions, polynomial functions and NonUniform Rational B-Splines (NURBS) functions are all commonly used deformation functions. 


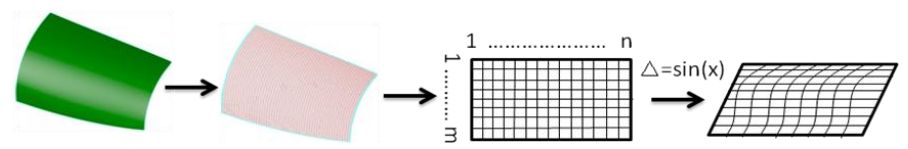

Figure 2. Schematic of surface deformation by LSF.

Here we give an example for LSF parametrization. It is mainly from the study from authors in reference [17]. Due to the symmetrical design along the longitude of the train, only one side of the symmetrical plane of the streamline is parameterized. As a result, the design parameters can be reduced by half. The parametric surfaces are separated into seven deformation regions, as shown in Figure 3(a). The nose drainage regions locate both sides of the streamline, originate from the nose tip and stretch backward to the end of the streamline. As shown in Figure 3(a), nose drainage is controlled by regions 3 and 6 , and a control point Point 1 is set here, extracting its y coordinate as the first design parameter $w_{1}$. The height of the nose tip is a crucial variable to aerodynamic performance of the train, so here we take it as a design variable for surface parametrization and call it nose height. As shown in Figure 3(a), the nose tip is completely controlled by regions 1 and 3 , and another control point Point2 is set here, extracting its $\mathrm{z}$ coordinate as the second design parameter $w_{2}$. The width of the streamline is namely the deformation of the streamline in $y$ direction, as shown in Figure 3(a), which is controlled by regions 4-7, and a control point Point 3 is set here, extracting its $\mathrm{y}$ coordinate as the third design parameter $w_{3}$. The slope of the cab window could be changed by surface deformation of region 5. However, in order to keep the deformed surface smooth, region 6 should be better included and deformed together with region 5. Consequently, the slope of the cab window will be controlled by regions 5 and 6 , and the fourth control point Point4 is set here, extracting its $\mathrm{z}$ coordinate as the fourth design parameter $w_{4}$. The shape of the cowcatcher is controlled by zone 2, and the fifth control point Point5 is set here, extracting its $\mathrm{x}$ coordinate as the fifth design parameter. For simplicity, all the deformation functions in this paper are trigonometric functions. Figure 3(b) shows the deformation of the nose and the cowcatcher. With use of the LSF method, surface deformation could be achieved by limited design variables. Meanwhile, transition between adjacent regions could be kept smooth during surface deformation, indicating that the LSF method could directly deform the concerned surface and keep highly efficient.

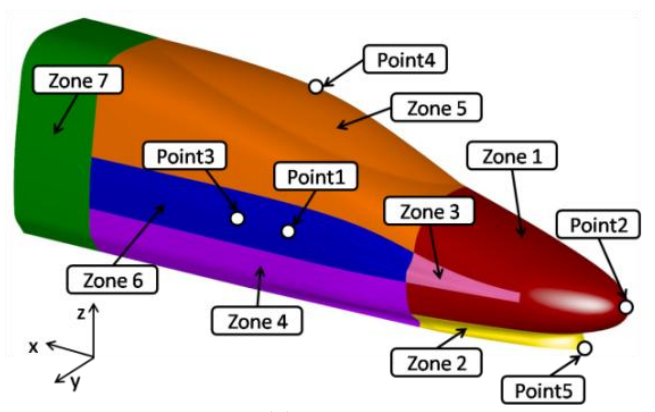

(a)

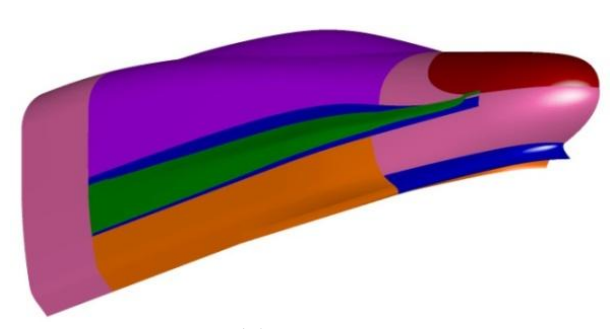

(b)

Figure 3. Deformation regions and local deformations: (a) Schematic of deformation regions; (b) deformation of the nose and cowcatcher

\subsection{FREE-FORM DEFORMATION METHOD}

FFD method was firstly proposed by Sederberg and Parry in 1986 [18]. It could be used for parametrization of twodimensional and three-dimensional shapes. It could still use simple operations to achieve deformations even for complex shapes. It is inspired by the physical phenomenon that the elastic body is deformed by external force. A control volume is built around the research object. Once the control volume is exerted an external force, all the shapes inside the volume would be deformed correspondingly, as well as the research object. The local coordinates of the research object will not change during the deformation. Consequently, a mapping relationship for the object before and after deformation could be established by the local coordinates. FFD method could be easily applied for parametrization of the shape entirely or locally.

Due to strong applicability of the FFD method, it is very suitable for parametrization of streamline shape of HSTs. Currently some commercial software such as SCULPTOR perform shape deformation on the base of this method. The FFD code was also developed in-house by the authors, aiming at parameterizing the practical streamline shape of HSTs.

A specific streamline shape is taken as an example. Due to symmetry, only half of the streamline shape is taken into consideration. As shown in Figure 4, the direction of length, width and height of the control volume are correspond to the $\mathrm{x}$, $\mathrm{y}, \mathrm{z}$ direction of the coordinate system. Eighteen control points are set along the $\mathrm{x}$ direction, while six points along $\mathrm{y}$ direction and five points along $\mathrm{z}$ direction. The total number of control points are 540. 

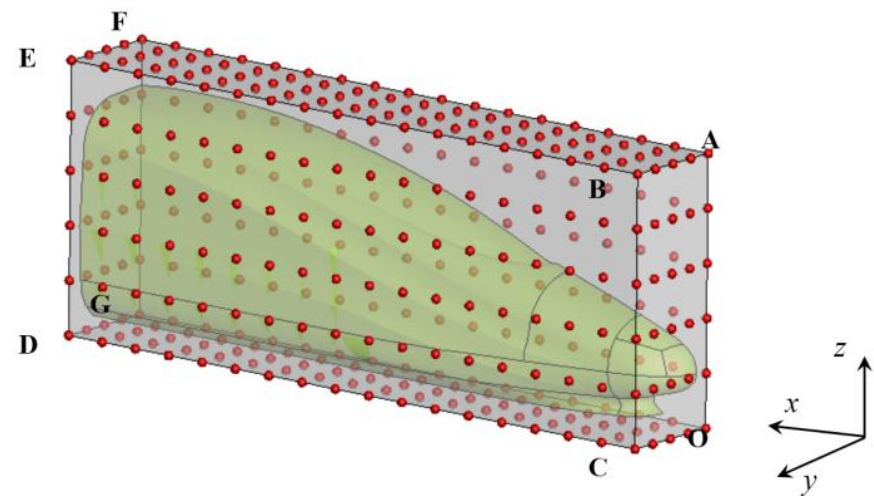

Figure 4. The FFD control volume and control points around the streamline shape

Although the number of control points is rather huge, the design variables should be chosen only for those with obvious physical significance and great influence on aerodynamic performance. According to the optimization design experience, the following control points are selected as design variables, as shown in Table 1.

Table 1. Design variables of the FFD method

\begin{tabular}{|c|c|c|c|}
\hline Number & Control Points & $\begin{array}{c}\text { Design } \\
\text { variables }\end{array}$ & Physical Meaning \\
\hline 1 & $(0,0,1)$ & $x$ & Nose Length \\
\hline 2 & $(0,0,1)$ & $z$ & Nose Height \\
\hline 3 & $(1,0,0)$ & $x$ & \multirow{2}{*}{$\begin{array}{l}\text { Bottom Length of the } \\
\text { Cowcatcher }\end{array}$} \\
\hline 4 & $(1,1,0)$ & $x$ & \\
\hline 5 & $(3,3,1)$ & $z$ & \multirow{2}{*}{$\begin{array}{l}\text { Entrance Height of the } \\
\text { Drainage }\end{array}$} \\
\hline 6 & $(3,3,2)$ & $z$ & \\
\hline 7 & $(3,4,0)$ & $y$ & \multirow{3}{*}{ Bottom Width } \\
\hline 8 & $(4,4,0)$ & $y$ & \\
\hline 9 & $(5,4,0)$ & $y$ & \\
\hline 10 & $(5,0,3)$ & $z$ & \multirow{2}{*}{$\begin{array}{c}\text { Height of the Driving } \\
\mathrm{cab}\end{array}$} \\
\hline 11 & $(6,0,3)$ & $z$ & \\
\hline 12 & $(6,3,2)$ & $z$ & \multirow{2}{*}{$\begin{array}{l}\text { Exit Height of the } \\
\text { Drainage }\end{array}$} \\
\hline 13 & $(9,4,2)$ & $z$ & \\
\hline 14 & $(13,4,1)$ & $y$ & \multirow{2}{*}{ Width of the Train Body } \\
\hline 15 & $(13,4,2)$ & $y$ & \\
\hline 16 & $(14,0,5)$ & $\mathrm{z}$ & Height of the Train Body \\
\hline
\end{tabular}

The design variables are mainly concerned about the nose, the cowcatcher, the drainage, the driving cab and the connection part between the streamline and the train body. The number of design variables is 16 , and it could be increased or decreased according to practical demands.

In order to validate the FFD method for streamline shape of HSTs, deformations on nose length, nose height and the height of the driving cab are performed. The shape comparisons before and after deformation are shown in Figure 5. It could be seen that FFD method could be efficiently used for deformation of the streamline shape and smooth deformation surfaces could be obtained.

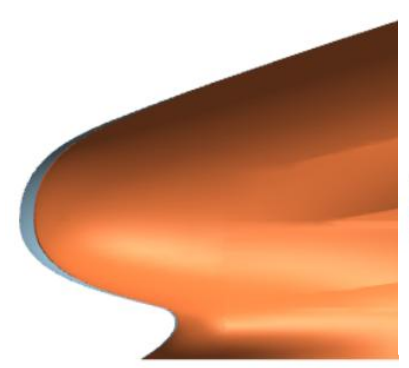

(a)

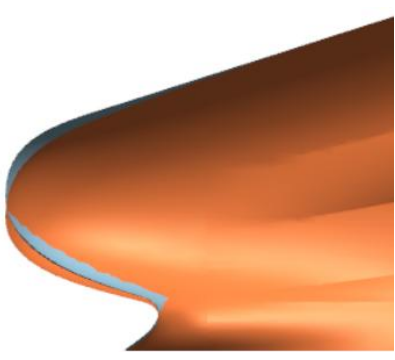

(b)

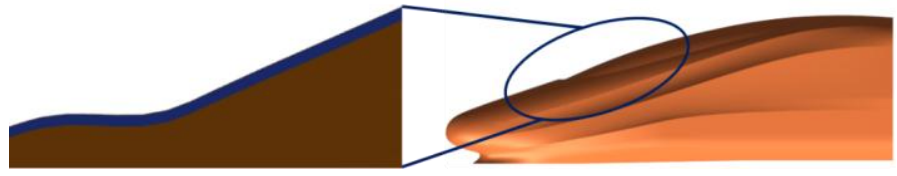

(c)

Figure 5. Shape comparison before and after deformation: (a) Nose Length; (b) Nose Height; (c) Height of the driving cab.

\subsection{METHODS COMPARISON AND ASSESSMENT}

The number of design variables for LSF method could be easily controlled by placing design variables on focused regions and smooth deformation could be ensured. LSF method owns the advantages of simple algorithm and strong popularization, and could be easily used to engineering problems. Compared to LSF method, FFD method is more applicable to parametrization of complex geometries, and only a proper control volume is needed together with control points with proper distribution. However, with the increase of control points, the number of design variables also increase, resulting in massive computational cost for aerodynamic shape optimization.

\section{SHAPE DESCRIPTION METHODS}

The same as section 2, the definition of shape description method is also given first. Based on systematically analysis on the shape of the object, key profiles or surface characteristics will be extracted. Considering the HST streamline, the profile of the longitudinal section is a key profile for the streamline. The maximum cross-sectional profile is another key profile as well. Once these profiles are determined, the basic shape of the streamline could be interpolated. These profiles could be categorized and described by certain curve equations, which have already been widely used in aeronautics and astronautics. For shape description methods, shape deformation could be achieved by varying the coefficients of the equations. Once the coefficients are changed, the streamline shape would be changed automatically for surface equation driven methods. For profile equations driven methods, the key profiles could also be altered, resulting in new deformed shape by spatial interpolation.

\subsection{MODIFIED VEHICLE MODELING FUNCTION METHOD}

The MVMF method is a profile equation driven method. As a result, key profiles along the streamline shape should firstly be analyzed. Figure 6 shows the key control profiles for the streamline shape, in which the longitudinal profile is controlled 
by L1, the longitudinal profile of the cowcatcher is controlled by $\mathrm{L} 2$, the horizontal profile and bottom profile are controlled by L3 and L4, and the maximum cross-sectional profile is controlled by L5. In general, the maximum cross-section is determined so as to match the existing train bodies. As a result, L5 will not be optimized and is given according to the maximum cross-section of CRH380A high speed trains. The profiles of L3 and L4 are similar and could be described with the same formula. L1 and L2 have the most influence on the nose shape. Totally different noses can be obtained via varying L1 and L2, especially the latter, which affects not only the aesthetic effect, but also the aerodynamic performance.

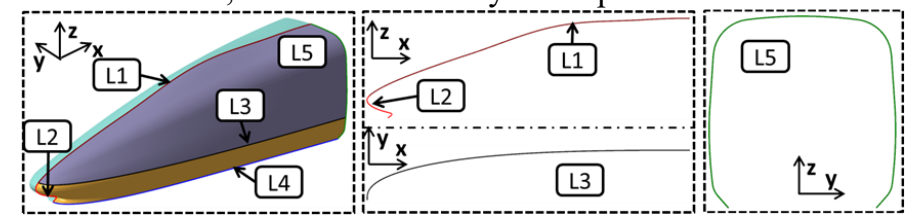

Figure 6. Key control profiles of the streamline shape

The Vehicle Modeling Function parametric method is derived by J.H. Rho etc. [19] through further improvement on Bernstein polynomial. This method can greatly reduce the design variables and better describe the profiles with little curvature in the shape design of automobiles. Yo-Cheon Ku etc. [20] introduced VMF method into the streamline shape design of HSTs and extracted four design variables to control the shape deformation. Although it is difficult to take the maximum crosssection and the volume of the nose into consideration through these variables and can hardly obtain any streamline shape that could be applied into actual application, it sheds light on the way to optimize the streamline shape of HSTs.

In reference [19], the basic form of two-dimensional profiles given by J.H. Rho is taken as:

$$
F\left(\frac{x}{c}\right)=\left(\frac{x}{c}\right)^{A_{1}}\left(1-\frac{x}{c}\right)^{A_{2}} S\left(\frac{x}{-}\right)+\left(1-\frac{x}{c}\right) Y+\left(\frac{x}{-}\right) Y
$$

In which $x$ ranges from 0 to $c \cdot Y_{1}$ and $Y_{2}$ are the vertical coordinates of the starting point and ending point, respectively. Different kinds of profiles can be obtained by changing the form of $S(x / c) . A_{1}$ and $A_{2}$ are the curvatures of the front and rear part of the profile. When $A_{1}$ ranges from 0 to 1 and $A_{2}$ is greater than 1 , the curvature at $\mathrm{x}=0$ for $F(x / c)$ tends to infinity, and at $\mathrm{x}=\mathrm{c}$ for $F(x / c)$ equals to 0 ; When $A_{2}$ ranges from 0 to 1 , the curvature at $\mathrm{x}=\mathrm{c}$ for $F(x / c)$ tends to infinity. Consequently, in order to avoid the infinite curvature at end points, cubic polynomial is adopted at the endpoints. The coefficients of the polynomial are determined by the coordinates and curvatures at the endpoints. When the curvature of the endpoints is finite values, in order to control the curvatures, proper revision is performed for above formula and takes the form as:

$$
F\left(\frac{x}{c}\right)=\left(\frac{x}{c}\right)^{A_{1}}\left(1-\frac{x}{c}\right)^{A_{2}} S\left(\frac{x}{c}\right)+G\left(\frac{x}{c}\right)
$$

$G(x / c)$ is used to control the change of curvatures at endpoints and typically takes the form of a polynomial, of which the coefficients are determined by the curvatures and coordinates at the end points.

Taking L1 as an example, the profile of L1 takes the form as:

$$
z(x)=\left(\frac{x-x_{11}}{x_{12}-x_{11}}\right)^{A_{11}}\left(1-\frac{x-x_{11}}{x_{12}-x_{11}}\right)^{A_{12}} a_{k 1}\left(1-\frac{x-x_{11}}{x_{12}-x_{11}}\right)^{a_{b 1}}+g(x)
$$

In which,

$$
g(x)=2\left(z_{12}-z_{11}\right) \frac{x-x_{11}}{x_{12}-x_{11}}-\left(z_{12}-z_{11}\right)\left(\frac{x-x_{11}}{x_{12}-x_{11}}\right)^{2}
$$

It is used to control the heights and curvatures at endpoints. $x_{11}$ and $x_{12}$ are the x-coordinates of the start point and end point. $z_{11}$ and $z_{12}$ are the $z$-coordinates of the start point and end point.

After obtaining the equations of key profiles, spatial interpolation will be performed between key profiles to obtain a basic streamline shape, and Laplacian smoothing method could be used for non-smooth regions. In order to better approximate the practical streamline, the superposition of the cab window and the lateral drainages will be conducted. Consequently, the final streamline shape will be obtained. Figure 7 shows the flow chart of MVMF for parametrization of the streamline shape of HSTs.

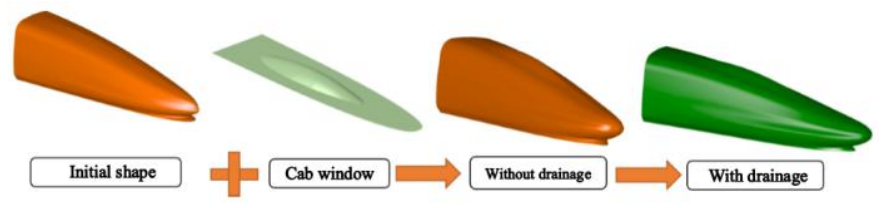

Figure 7. Flow chart of MVMF for parametrization of the streamline shape of HSTs

Figure 8 shows different streamline shapes of HSTs. It can be seen that totally different streamlines can be obtained by adjusting the design variables. The basic outline of the streamline shape could be sketched by three profiles. The profile of the cowcatcher zone greatly enriches the streamline shape that could be further adjusted by the shape of the cab window and the drainage.

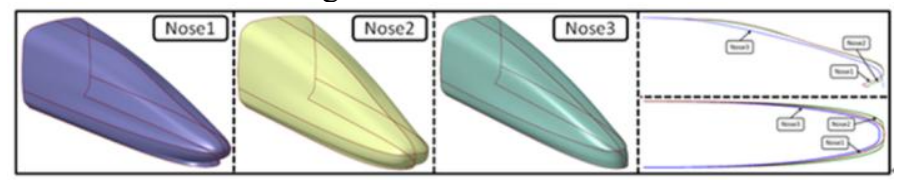


Figure 8. Different streamlines of HSTs

\subsection{CLASS FUNCTION/SHAPE FUNCTION TRANSFORMATION METHOD}

CST method was firstly proposed by Kulfan and Bussoletti in 2006 [21]. A class function and a shape function are utilized to represent the profile of airfoils. This method is widely used in parametrization of aeronautics and astronautics vehicles, and seldom references could be referred to for its application to the parametrization of streamline shape of HSTs. Based on the characteristics of the streamline shape, the authors developed the codes of CST method in-house, and determined some key values of coefficients in class function and shape function to be properly used for streamline shapes. In order to approximate closely to the practical geometry, the optimal CST method has been developed based on optimization algorithms.

For three-dimensional CST parametrization, based on Bernstein polynomials, the cross-sectional expression could be determined, taking the form as:

$$
\zeta(\psi)=C_{N 2}^{N 1}(\psi) \sum_{i=0}^{n} b_{i} B_{n}^{i}(\psi)+\Delta \zeta_{N}(\psi)
$$

Where $\psi=y / L_{w}$, which is the normalized length in the cross section. $L_{w}$ is the length of the cross-sectional profile. $\zeta(\psi)$ is the height of the profile at the position $\psi$. $C_{N 2}^{N 1}(\psi)$ is the class function, where $\mathrm{N} 1$ and $\mathrm{N} 2$ are the coefficients which define the basic profile type. $\sum_{i=0}^{n} b_{i} B_{n}^{i}(\psi)$ is the shape function, which could make local or overall adjustment on the base of the basic profile by varying the coefficient $b_{i}$ until the final profile is obtained. $b_{i}, i=0,1, \cdots, n$, are the weighting factors of Bernstein polynomials. $B_{n}^{i}(\psi)$ is the $\mathrm{i}$-th basis function of $\mathrm{N}$-th order Bernstein polynomials. $\Delta \zeta_{N}(\psi)$ is the offset at the position $\psi$.

The three-dimensional surface could be considered as the combination of a series of cross-sections along the axial direction. Different locations in axial direction could be described by the shape function. As a result, the expression of the whole surface could be obtained. Similarly, we define $b_{i}$ :

$$
\begin{gathered}
b_{i}=C_{M 2}^{M 1}(\eta) \bullet S(\eta)+\Delta \zeta_{M}(\eta) \\
S(\eta)=\sum^{m} b_{j} N_{k}^{j}(\eta)
\end{gathered}
$$

Where $\eta=x / L$ is $L$ is the axial length of the geometry. Taking $b_{i}$ into the definition of $\varsigma(\psi)$ :

$$
\zeta(\psi, \eta)=C_{N 2}^{N 1}(\psi) C_{M 2}^{M 1}(\eta) \sum_{i=0}^{n} \sum_{j=0}^{m} b_{i, j} N_{k}^{i}(\psi) N_{k}^{j}(\eta)+\Delta \zeta_{M, N}(\psi, \eta)
$$

Meanwhile, the boundary profile for the projection plane of $\psi$ and $\eta$ should be parameterized. That is,

$$
Z(\eta)=C_{T 2}^{T 1}(\eta) \sum_{i=0}^{w} b_{t} N_{k}^{i t}(\psi)
$$

The above formulas define an analytical description for a three-dimensional surface.

Considering that the downward part and backward part of the streamline shape are completely open, the coefficient M2 and T2 should be 0 . Besides, the streamline shape is symmetrical bilaterally, which requires that $\mathrm{N} 1$ and N2 should be equal to each other and the weight coefficient matrix should be symmetrical too. Meanwhile, in order to further reduce the number of design variables, the orders of shape functions in three dimensions are all 3.

After simplification, the number of design variables for CST parametrization of a streamline shape is 15 , which includes three coefficients for class functions, four independent weight coefficients in shape functions and eight independent coefficients in weight coefficient matrix. All the design variables are listed in Table 2.

Table 2. Design variables in 3d-CST parametrization for a streamline shape

\begin{tabular}{c|cc}
\hline $\begin{array}{c}\text { coefficients for class } \\
\text { functions }\end{array}$ & $\mathrm{N} 1, \quad \mathrm{M} 1, \quad \mathrm{~T} 1$ \\
\hline $\begin{array}{c}\text { weight coefficients in shape } \\
\text { functions } b_{t}\end{array}$ & $\mathrm{bt}(0), \quad \mathrm{bt}(1), \quad \mathrm{bt}(2), \quad \mathrm{bt}(3)$ \\
\hline $\begin{array}{c}\text { coefficients in weight } \\
\text { coefficient matrix } b_{w}\end{array}$ & $\begin{array}{l}\mathrm{bw}(0,0), \\
\mathrm{bw}(1,0),\end{array} \mathrm{bw}(0,1), \quad \mathrm{bw}(1,1), \quad \mathrm{bw}(1,2), \quad \mathrm{bw}(0,3)$ & $\mathrm{bw}(1,3)$ \\
\hline
\end{tabular}

Different streamline shapes could be obtained by justifying the values of design variables. Figure 9 show the different streamline shapes.

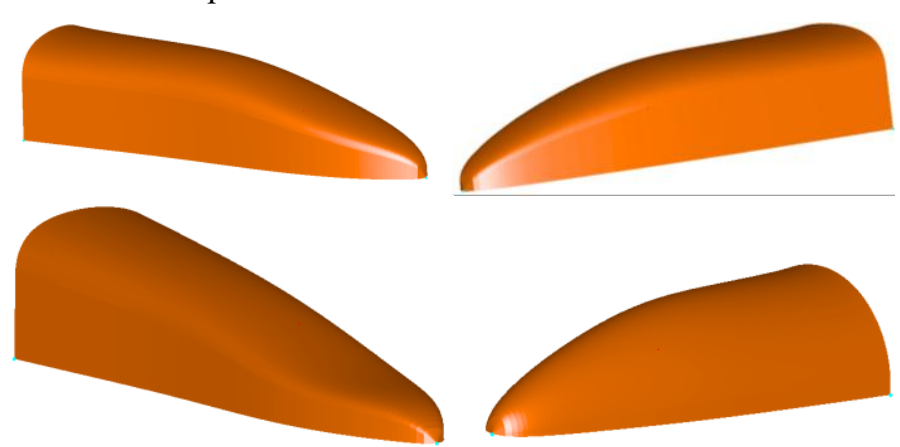

Figure 9. Different streamline shapes by CST parametrization

It could be seen that the streamline shape could be given randomly by the 15 design variables. However, sometimes we want to obtain an initial shape of a practical streamline shape and perform aerodynamic optimization from the practical shape, which requires that the CST method could approximate close to 
the practical shape. As a result, the optimal CST method is developed on the base of optimization algorithms. The exact process could be described as follows: Firstly, structural grids should be generated along the surface of practical streamline shape and make sure the grid distribution in $\mathrm{x}$ and $\mathrm{y}$ direction to be equal-distant for adjacent grids. Secondly, calculating the difference between $Z(\eta, \psi)$ and the practical value. Thirdly, loop through all $\eta$ and $\psi$, and summarize all the differences. In the end, minimize the summation of all differences by optimization algorithms and get the optimal values of design variables.
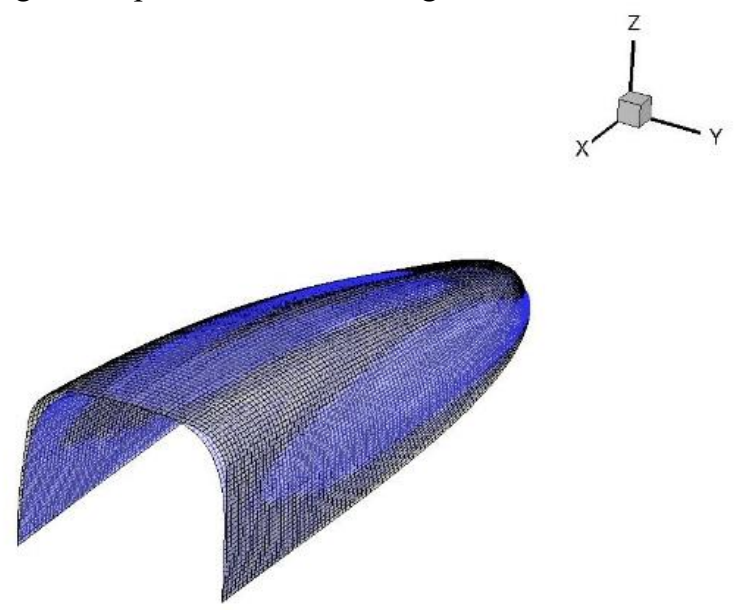

Figure 10. Comparison of an optimized CST shape and the practical shape

Figure 10 shows the comparison of an optimized CST shape and the practical shape. It can be seen that most places are close to each other for the optimized CST shape and the practical one, although slighter big difference could be found locally.

\subsection{METHODS COMPARISON AND ASSESSMENT}

MVMF method is a parametrization approach driven by equations of key profiles, and could be precisely describe the three-dimensional streamline shape by use of spatial interpolation technique. This method could be used for conceptual design of streamline shape and the aerodynamic optimization of a practical streamline as well. The design efficiency of the streamline shape could be greatly improved by this method. However, the design variables get poor physical significance, since all design variables are defined by the coefficients of key profiles.

Compared to MVMF method, CST method is driven by equations of key surfaces. This method owns strong integrity, since no spatial interpolation is needed. Consequently, the smoothness of the CST surface is better than MVMF surface. Meanwhile, CST method suffers the same with MVMF method in less physical significance of design variables.

\section{CONCLUSIONS}

In the present paper, the authors systematically introduce a series of work on parametrization of streamline shape of HSTs.
Four parametrization methods are presented, which are LSF method, FFD method, MVMF method and CST method. The algorithms and whole processes are all introduced, too. By method innovation and extension, these four methods are all successfully applied into streamline shape parametrization. Comparison study shows that LSF and FFD both benefit from simple algorithms and strong generality. The design variables for LSF and FFD own very intuitive physical meanings. However, the number of design variables for LSF could be easily controlled while difficult for FFD when the complexity of geometry increases. MVMF and CST are both suitable for conceptual design of streamline shape and for the aerodynamic optimization of a practical streamline. The design variables for both methods come from the coefficients of key profile equations or surface equations. As a result, they both suffer from the lack of physical significance. Generally speaking, the authors' work provides abundant choices for parametrization of streamline shape of HSTs. The parametrization method could be chosen automatically according to specific physical background.

\section{ACKNOWLEDGMENTS}

This work was supported by Advanced Rail Transportation Special Plan in National Key R\&D Program of China under 2016YFB1200601-B13. And Computing Facility for Computational Mechanics Institute of Mechanics, Chinese Academy of Sciences is gratefully acknowledged.

\section{REFERENCES}

[1] Raghuathan S, Kim H D, Setoguchi T Aerodynamics of high-speed railway train.[J], Progress in Aerospace Sciences, 2002, 8(6): 469-514

[2] Joseph A S. Aerodynamics of High-speed Trains[J]. Annual Reviews Fluid Mechanics, 2001, 33(1):371-414.

[3] Yang Guowei, Guo Dilong, Yao Shuanbao, et al. Aerodynamic design for China new high-speed trains. Science China Technological Sciences, 2012, 55(7): 1923-1928.

[4] Hassan Hemida, Sinisa Krajnovic, LES study of the influence of the nose shape and yaw angles on flow structures around trains, J. Wind Eng. Ind. Aerodyn. 98(2010):34-46.

[5] SUN Zhenxu, SONG Jingjing, AN Yiran, Optimization of the head shape of the CRH3 high speed train, SCIENCE CHINA, 2010, 12: 3356-3364.

[6] Yo-Cheon Ku, Joo-Hyun Rho, Su-Hwan, etc. Optimal cross-sectional area distribution of a high-speed train nose to minimize the tunnel micro-pressure wave, Struct Multidisc Optim, 2010, 42: 965-976

[7] V. V. Vytla, P. G. Huang, R. C. Penmetsa, Multi objective aerodynamic shape optimization of high speed train nose using adaptive surrogate model, AIAA-2010-4383.

[8] Ku Y-C, Kwak M-H, Park H-I, Lee D-H, Multiobjective optimization of high-speed train nose shape using the vehicle modeling function. In: 48th AIAA aerospace sciences meeting, 2010, Orlando, USA. 
[9] Krajnovic S. Shape optimization of high-speed trains for improved aerodynamic performance. P I Mech Eng F-J Rai, 2009, 223: 439-452.

[10] Xiong J, Li T, Zhang J Y. Shape optimization of highspeed trains under multi runing conditions (in Chinese). Sci Sin Tech, 2016, 46: 313-322

[11] Li M, Li M G, Li G Q, Kong F B, Liu B, optimized design of parametric-driven aerodynamic shape of high-speed EMU head-type (in Chinese), Journal of the China Railway Society, 2013, 35(11): 14-20

[12] Zhang L, Zhang J Y, Li T, Zhang W H, Multiobjective aerodynamic optimization design for head-shape of high speed trains (in Chinese), Journal of Southwest Jiaotong University, 2016, 51(6): 1055-1063

[13] Li M, Liu B, Zhang L, Optimization Design on Key Structural Parameter of High Speed EMU Head-type Aerodynamic Shape (in Chinese), Journal of mechanical engineering, 2016, 52(20):120-125

[14] Li R, Xu P, Peng Y and Li P, Multi-objective optimization of a high-speed train head based on the FFD method, Journal of Wind Engineering and Industrial Aerodynamics, 2016, 152: 41-49

[15] Muñoz-Paniagua, J., García, J., Crespo, A., Genetically aerodynamic optimization of the nose shape of a high-speed train entering a tunnel. Journal of Wind Engineering and Industrial Aerodynamics, 2014, 130: 48-61.

[16] Iida M, Matsumura T, Nakatani K, et al. Effective nose shape for reducing tunnel sonic boom[J]. QR of RTRI, 1997, 4(38): 206-211.

[17] Yao Shuanbao, Guo Dilong, Sun Zhenxu, et al. Multiobjective optimization of the streamline head of high-speed trains based on the Kriging model, Science China Technological Sciences, 2012, 55(12): 3494-3508.

[18] Sederberg, T. W., Parry, S. R., Free-Form Deformation of Solid Geometric Models, Computer Graphics, 1986, 20 (4): 151-159.

[19] J.H. Rho, Y.C. Ku, S.H. Yun et al. Development of Vehicle Modeling Function for 3-Dimensional Shape Optimization, Journal of Mechanical Design, 2009, 131(12): 121004-1-10.

[20] Ku Y-C, Kwak M-H, Park H-I, Lee D-H, Multiobjective optimization of high-speed train nose shape using the vehicle modeling function. In: 48th AIAA aerospace sciences meeting, 2010, Orlando, USA

[21] Kulfan BM., Bussoletti J.E.. Fundamental Parametric Geometry Representations for Aircraft Component Shapes, 11th AIAA/ISSMO Multidisciplinary Analysis and Optimization Conference, Portsmouth, VA, 2006: 1-42. 